\title{
Weise, Marion
}

\section{Es ist noch jemand mit uns hier. Puppet-Interviews in der Forschung mit} Kindern

Hedderich, Ingeborg [Hrsg.]; Reppin, Jeanne [Hrsg.]; Butschi, Corinne [Hrsg.]: Perspektiven auf Vielfalt in der frühen Kindheit. Mit Kindern Diversität erforschen. 2., durchgesehene Auflage. Bad Heilbrunn : Verlag Julius Klinkhardt 2021, S. 158-171

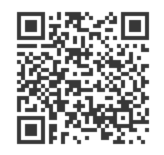

Quellenangabe/ Reference:

Weise, Marion: Es ist noch jemand mit uns hier. Puppet-Interviews in der Forschung mit Kindern - In: Hedderich, Ingeborg [Hrsg.]; Reppin, Jeanne [Hrsg.]; Butschi, Corinne [Hrsg.]: Perspektiven auf Vielfalt in der frühen Kindheit. Mit Kindern Diversität erforschen. 2., durchgesehene Auflage. Bad Heilbrunn : Verlag Julius Klinkhardt 2021, S. 158-171 - URN: urn:nbn:de:0111-pedocs-222539 - DOI: 10.25656/01:22253

https://nbn-resolving.org/urn:nbn:de:0111-pedocs-222539

https://doi.org/10.25656/01:22253

in Kooperation mit / in cooperation with:

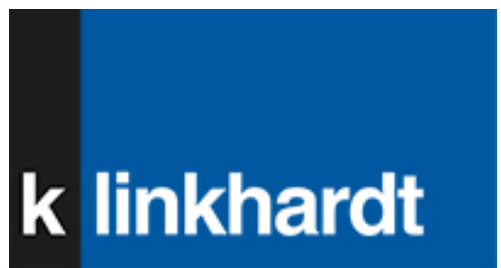

http://www.klinkhardt.de

\section{Nutzungsbedingungen}

Dieses Dokument steht unter folgender Creative Commons-Lizenz: http://creativecommons.org/licenses/by-nc-nd/4.0/deed.de - Sie dürfen das Werk bzw. den Inhalt unter folgenden Bedingungen vervielfältigen, verbreiten und öffentlich zugänglich machen: Sie müssen den Namen des Autors/Rechteinhabers in der von ihm festgelegten Weise nennen. Dieses Werk bzw. dieser Inhalt darf nicht für kommerzielle Zwecke verwendet werden und es darf nicht bearbeitet, abgewandelt oder in anderer Weise verändert werden.

Mit der Verwendung dieses Dokuments erkennen Sie die Nutzungsbedingungen an.

\section{Terms of use}

This document is published under following Creative Commons-License: http://creativecommons.org/licenses/by-nc-nd/4.0/deed.en - You may copy, distribute and transmit, adapt or exhibit the work in the public as long as you attribute the work in the manner specified by the author or licensor. You are not allowed to make commercial use of the work or its contents. You are not allowed to alter, transform, or change this work in any other way.

By using this particular document, you accept the above-stated conditions of use.

\section{Kontakt / Contact:}

\section{peDOCS}

DIPF | Leibniz-Institut für Bildungsforschung und Bildungsinformation

Informationszentrum (IZ) Bildung

E-Mail: pedocs@dipf.de

Internet: www.pedocs.de

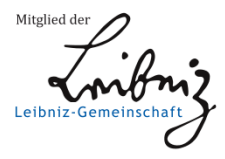




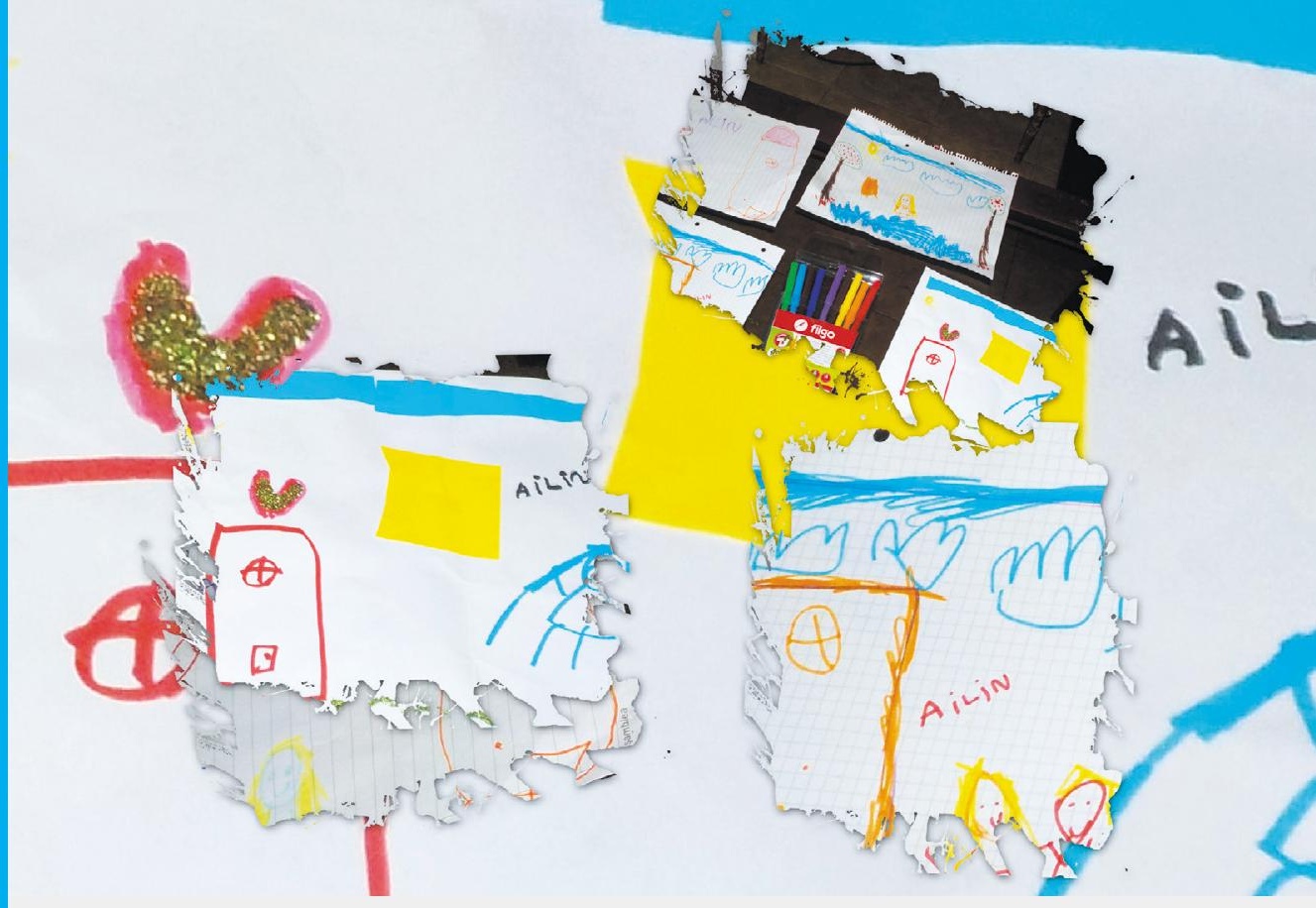

Ingeborg Hedderich

Jeanne Reppin

Corinne Butschi

(Hrsg.)

\section{Perspektiven auf Vielfalt in der frühen Kindheit}

Mit Kindern Diversität erforschen

2. Auflage 


\section{Ingeborg Hedderich Jeanne Reppin \\ Corinne Butschi \\ (Hrsg.)}

\section{Perspektiven auf Vielfalt in der frühen Kindheit}

Mit Kindern Diversität erforschen

2., durchgesehene Auflage 
Dieser Titel wurde in das Programm des Verlages mittels eines Peer-Review-Verfahrens aufgenommen. Für weitere Informationen siehe www.klinkhardt.de.

Bibliografische Information der Deutschen Nationalbibliothek Die Deutsche Nationalbibliothek verzeichnet diese Publikation in der Deutschen Nationalbibliografie; detaillierte bibliografische Daten sind im Internet abrufbar über http://dnb.d-nb.de.

2021.g. () by Julius Klinkhardt.

Foto Umschlagseite 1: Ailin, 5 Jahre, Provinz Entre-Rios, Argentinien und Corinne Butschi.

Druck und Bindung: Bookstation GmbH, Anzing.

Printed in Germany 2021.

Gedruckt auf chlorfrei gebleichtem alterungsbeständigem Papier.

(c) (1) $\Theta(D i e$ Publikation (mit Ausnahme aller Fotos, Grafiken und Abbildungen) ist veröffentlicht unter der Creative Commons-Lizenz: CC BY-NC-ND 4.0 International https://creativecommons.org/licenses/by-nc-nd/4.0/ 


\section{Inhalt}

Perspektiven auf Vielfalt in der frühen Kindheit.

Mit Kindern Diversität erforschen

Einleitung

Ingeborg Hedderich, Jeanne Reppin und Corinne Butschi

\section{Theorie}

Kindheit und Kindheitsforschung im Wandel

Corinne Butschi und Ingeborg Hedderich.

Erziehungswissenschaftliche Perspektiven auf Vielfalt, Heterogenität,

Diversity/Diversität, Intersektionalität

Katharina Walgenbach.

Vorderbühne - Hinterbühne. Zur Interdependenz der Horizonte von Diversität und Gleichheit

Cornelie Dietrich. 60

Differenzen und die Heterogenität von Kindern -

Einsätze blicktheoretischer Forschung

Friederike Schmidt.

\section{Methodologie und Methoden}

Kindgerecht forschen. Ein Überblick

Corinne Butschi und Ingeborg Hedderich.

Ankerpunkte, Wegmarken und Herausforderungen einer ethischen Forschung mit Kindern

Jeanne Reppin

Mit Kindern Interviews führen: Ein praxisorientierter Überblick

Susanne Vogl

Es ist noch jemand mit uns hier.

Puppet-Interviews in der Forschung mit Kindern

Marion Weise

Forschungsmethodische Vielfalt. Der Mosaic Approach

Sandra Schütz und Eva Theresa Böhm

Warum eigentlich? Philosophieren mit jungen Kindern

Martina Bernasconi. 


\section{Forschungszusammenhänge und Praxisreflexionen}

Ethnologische Kindheitsforschung im Überblick mit besonderer

Berücksichtigung der teilnehmenden Beobachtung

Werner M. Egli.....

Diversitätsbewusstes Denken und Handeln in Kindertageseinrichtungen -

Forschen mit Interviews und Interviewstreifzügen

Steffen Brockmann

Zur Relevanz der Zurechnung von Komplexität. Das Interview als

Methode der Datenerhebung im Alter früher Kindheit im Kontext von Behinderung

Martina Kaack.

Kamerakids: Forschen mit Photovoice

Corinne Butschi, Melike Hocaoglu, Manuel Zanardini, Valentin Mettler,

Ana Luisa Baumann-Santiago Martínez, Guillermina Chabrillon und

Ingeborg Hedderich.

Vielfalt in internationaler Zusammenarbeit erforschen. Ein Dialog

Corinne Butschi und Guillermina Chabrillon....

Einblick in ein vielfältiges Tätigkeitsfeld frühkindlicher Förderung in

Argentinien

Barbara Schoch

Das Erleben von Kindern mit Fluchterfahrung.

Forschen mit non-verbalen Zugängen

Marion Weise, Marion Lempp und Regine Morys

Wie erleben platzierte Vorschulkinder die Zugehörigkeit zu ihren

komplexen Beziehungswelten?

Forschen mit dem Geschichtenstammverfahren der

MacArthur Story Stem Battery

Maria Mögel.....

Lebenswelt Gemeinde: Mit Kindern forschen -

Aus Perspektiven und Methoden ein Mosaik zusammenfügen

Gabriela Muri und Heidi Simoni

Vom Schweizer Kindergarten ins Außerschulische, nach Ghana, und wieder zurück: Wenn Kinder und eine Ethnografin gemeinsam

,Grenzen' überschreiten

Ursina Jaeger

Die Autor*innen 


\section{Marion Weise}

\section{Es ist noch jemand mit uns hier. Puppet-Interviews in der Forschung mit Kindern}

\section{Einleitung}

"Grown-ups don't really know what it is like to be a child in a day care. We children know. That's why I have to tell you about it." (Eide \& Winger 2005, 86)

Das Interesse an der Perspektive der Kinder, der Anerkennung dieser Expertengruppe als aktiv Gestaltende und „Seiende“ (Qvortrup 1994 zitiert nach Honig 1999, 33) als auch aus dem Wissen um die Konstruktionen und Reproduktionen von heterogenen Kindheiten, machen es unabdinglich explizit mit Kindern zu forschen und geeignete Zugänge zu diskutieren, die ihre Stimme zur Geltung bringen (vgl. Honig 1999; Roux 2002; Nentwig-Gesemann, Walther \& Thedinga 2017).

Vor diesem Hintergrund wird in der Kindheitsforschung kritisch diskutiert, ob sie besondere Zugangsmöglichkeiten benötigt, die auf die speziellen Bedürfnisse und Bedingungen der Kinder zugeschnitten sind. Es wird davon ausgegangen, dass Forschungsmethoden nicht einfach unhinterfragt in die Kindheitsforschung importiert werden können (vgl. Mey 2003; Paus-Hasebrink 2017). Autoren wie Trautmann (2009) oder Vogl (2015) argumentieren, dass aufgrund unterschiedlicher Kompetenzen von Kindern spezielle Methoden entwickelt werden müssen.

Diese Argumentation erfolgt tendenziell aus einer erwachsenzentrierten Sichtweise, sie geht davon aus, dass Kinder bestimmte Kompetenzen wie beispielsweise kohärentes Erzählen, Perspektivenübernahme und Langzeiterinnerungsvermögen erst in rudimentärer Form besitzen oder entwickeln und es daher spezifische Methoden braucht, um diesem Entwicklungsstand entgegen zu kommen und auszugleichen. Autor*innen wie Groundwater-Smith, Dockett \& Bottrell (2014) und Punch (2002) vertreten vielmehr die Ansicht, dass die Methode generell angemessen sein muss und dies als Desiderat für alle Forschungsprojekte und Designs mit allen Zielgruppen gelte (vgl. Groundwater-Smith, Dockett \& Bottrell 2014; Punch 2002) Sie argumentieren für eine individuell personenzentrierte Anpassung anstelle einer auf Kinder angepasste Spezifika, wobei es abzusichern gilt, dass die Methoden die Würde und Integrität des Gegenübers wahren und den 
vielfältigen Ausdrucksarten des Erzählens gerecht werden (vgl. ebd.; Clark 2017; Nentwig-Gesemann et al. 2017).

Diesem Grundgedanken folgt dieser Beitrag, fasst Kinder als Konstruierende und aktive Subjekte im Forschungsprozess, wobei die Zielgruppe in der Beforschung dennoch besondere Berücksichtigung von Seiten des erwachsenen Forschenden bedarf. Kinder stellen aufgrund der Zuweisungen in der generationalen Ordnung und ihrer ,anthropologischen Angewiesenheit auf Responsivität, Pflege und Schutz" (Andresen, Koch \& König 2015, 8) eine vulnerable Gruppe dar und die Verletzlichkeit darf durch die „Akteurszentrierung“ (ebd.) in der Kindheitsforschung nicht ausgeblendet werden. Es bedarf u.a. in der Forschung mit Kindern einer Ethik, die beständig im Prozess überprüft, ob die Methode und das Vorgehen für alle beteiligten Akteure passend und angemessen ist (vgl. Phelan \& Kinsella 2013; Powell, Graham \& Truscott 2016) und ihren je spezifischen gewählten Zugang reflektiert.

Dieser Beitrag stellt das Puppet Interview als einen Zugang zur Befragung von Kindern im Alter von drei- bis sechs Jahren vor. Zuerst wird die Bedeutung der Puppe im Spiel für Kinder in diesem Altersbereich knapp skizziert. Überblicksartig soll anschließend dargestellt werden, wie Handpuppen bereits in Forschungskontexten eingesetzt werden, um daran anknüpfend die spezifische Methode des Puppet Interviews, wie diese von der Autorin weiter in der Forschungspraxis spezifiziert und angewandt wurde, darzustellen. Dabei werden in einer kritischen Reflexion Stärken sowie Schwächen und Herausforderungen dieser Methode herausgearbeitet. Zudem beinhaltet der Beitrag reflexive Überlegungen, wie diesen adäquat begegnet werden kann beziehungsweise wie diese Berücksichtigung in der konkreten methodischen Umsetzung finden können.

\section{Bedeutung der Handpuppe für Kinder im Spiel}

Die Puppe stellt ein vertrautes Spielobjekt für Kinder dar und ist eines der ältesten und bedeutendsten Spielzeuge (vgl. Pohl 2014; Gauda 2001). Im Kleinkindalter ist die Puppe oder das Schmusetier ein bedeutendes Übergangsobjekt, das durch seine Anwesenheit Sicherheit und den Kontakt zur Umwelt ermöglicht, in dem es einen Zwischenbereich zwischen dem Innen- und der Außenwelt eröffnet. Später kann die Puppe im Rollenspiel zur Auseinandersetzung mit verschiedenen Rollenmuster und Scripts zur Bearbeitung von bedeutsamen Thematiken als auch zur Entwicklung der eigenen Identität dienen (vgl. Oerter 1999). Puppen haben für Kinder eine magische Ausstrahlung und sind laut Gauda „ein Abbild des Menschen “ $(2001,10)$, sie schärfen als Gegenüber die Möglichkeit zur Empathiefähigkeit (vgl. Pohl 2014). Dies wird besonders deutlich, wenn man Kinder beim Spiel mit Puppen beobachtet. Ungeachtet der Herkunft und der Zeitperiode, instinktiv 
und ganz natürlich wird die Puppe zum „Gegenüber für das Kind“ (Gauda 2001, 10). Die Puppe ist allerdings nicht denkbar ohne den Spieler oder die Spielerin, er oder sie erweckt sie zum Leben und ohne ihn oder sie bleibt die Puppe funktionslos (vgl. Pohl 2014).

Aufgrund der Beobachtungen von Spiel und Rollenspiel von Kindern auf Spielplätzen entwickelte Jacob Levy Moreno das Psychodrama, das die Möglichkeit eröffnet, Gefühle, Empfindungen, Ängste auf der „äußeren Bühne“ (Gauda 2001, 10) auszuspielen und damit laut zu machen. Er konnte feststellen, dass es Kindern im direkten Gespräch schwer fällt, Gefühlen und Ängsten Ausdruck zu verleihen, nicht aber, wenn dies über die Puppe geschieht. Bereits seit den 20er Jahren werden Puppen systematisch in der Therapie mit Kindern eingesetzt.

\section{Der Einsatz von Handpuppen in forschenden Kontexten}

Aus der Bedeutung von Puppen für Kinder im Rollenspiel und damit für die kindliche Entwicklung, liegt es nahe gerade Handpuppen als Werkzeug der Forschung zu verwenden, um damit eine besonders geeignete und angemessene Zugangsweise zu Kindern zu ermöglichen. In der Kindertherapie hat sich die Verwendung von Puppen und Handpuppen bereits etabliert, für die Kindheitsforschung in nicht-therapeutischen und tiefenpsychologischen Kontexten stellt sie noch eine neuere Entwicklung dar.

„Although puppets have been employed by various disciplines in clinical and community (e.g. homes and schools) environments, little has been written about their use as a communication tool in research" (Epstein, Stevens, McKeever, Baruchel \& Jones 2008, 49).

Im englischsprachigen Raum wird die Puppe vor allem in quantitativ orientierten Designs der Entwicklungspsychologie verwendet. Roth, Daads and McAllon (2004) entwickelten mit den Handpuppen ein Verfahren, um Einschätzungen und Positionen von Kindern auf einer Likert-Skala zu erhalten ohne hierbei auf Schrift zurückgreifen zu müssen. Die Kinder wurden mit zwei gegensatzpaarigen Aussagen konfrontiert, die durch zwei unterschiedliche Handpuppen, in diesem Fall Teddybären, vertreten wurden. Die Kinder sollten den Teddybär wählen, dem sie am ehesten zustimmen bzw. mit dem sie am ehesten übereinstimmen. Dieses so genannte forced-choice Format ist darauf ausgerichtet, quantitative Ergebnisse zu erzielen (vgl. Roth, Dadds \& McAloon 2004).

Das bekannteste Verfahren, in dem Handpuppen zum Einsatz kommen, ist das Berkeley Puppet Interview (BPI), welches 1998 von Measelle, Ablow, Cowan \& Cowan eingesetzt und publiziert wurde und sich seither über den englischsprachigen Raum hinaus als valides Instrument etablieren konnte. Hierbei handelt 
es sich um einen semi-strukturierten Interviewtypus, der die Wahrnehmung von Kindern im Alter von vier bis acht Jahren zu ihrem Familienumfeld, zum schulischen Umfeld sowie das Verhältnis zu den Sozialinstanzen misst. Dabei werden zwei Handpuppen eingesetzt, die divergierende Positionen vertreten, und das Kind kann dann erzählen, wie es sich selbst und seine Umgebung zu dem durch die Puppen eingegrenzten Gegenstand wahrnimmt (vgl. Perren, Stadelmann \& von Klitzing 2009; Perren \& von Klitzing 2008). Dieses Vorgehen unterscheidet sich damit von den forced-choice Formaten, insofern das Kind hier die Möglichkeit hat, in der natürlichen Kommunikation und Interaktion mit den Handpuppen die eigene Sichtweise auszuführen. Die Mehrheit der Kinder antworten nach Angaben der Autor*innen ausführlich verbal, aber auch performatives Antwortverhalten kann durch den BPI und dessen Codierungssystem erfasst werden. Dieses wird durch geschulte Kodierer mittels Videos analysiert, dabei werden die teils komplexen Antworten der Kinder entschlüsselt und auf einer Likert-ähnlichen siebenstufigen Skala eingeschätzt (vgl. Measelle, Ablow/, Cowan \& Cowan 1998). Die in diesem Beitrag verwendete Methode des Handpuppeninterviews oder Puppet Interviews wird von den forced-choice Formaten als auch von dem BPI abgegrenzt. Die Puppe ist prädestiniert dafür, eine detaillierte Innenperspektive zu ermöglichen und damit einen qualitativen Zugang zu den subjektiven Deutungsstrukturen der Kinder herzustellen. Die bisher vorgestellten Möglichkeiten nutzen die Stärke der Methodik bisher vor allem in quantitativen Designs. Die Autorin lehnt sich daher an die Grundlagenarbeit von Paus-Haase [Hasebrink] (1998) und Lampert (2000) an, die das Handpuppeninterview in medienpädagogischen Kontexten zur Generierung qualitativer Theorien verwendeten. In der von Weise $(2008 ; 2012)$ weiter modifizierten und in internationalen Settings erprobten Methode spricht die Interviewerin oder der Interviewer quasi durch die Puppe. Die interviewende Person nutzt diese als Mediator*in zwischen sich und dem Kind.

Die Verwendung der Handpuppe als Hilfsmittel in Methoden der Kindheitsforschung findet bereits mehrfach Erwähnung (vgl. Paus-Haase 1998; Lampert 2000; Fuhs \& Schneider 2012; Marci-Boehncke \& Rath 2014), die zu berücksichtigenden Schritte im Forschungsprozess, sowie die Vor- und Nachteile eines solchen Instrumentes sind bisher noch marginal reflektiert. Diese müssen vor dem Hintergrund der Herstellungsleistung von Kindern und Kindheiten über Dokumente und Methoden (vgl. Betz \& Bischoff 2013; Betz \& Eßer 2016) auf die „Bezogenheit von erwachsenen Forschenden und beforschten Kindern“ (Mey 2003, 22) hin methodologisch diskutiert und reflektiert werden. 


\section{Methode Puppet Interview in der Forschungspraxis}

\subsection{Die Auswahl der Handpuppe als einen zentralen methodischen Schritt planen}

Die Auswahl einer Puppe und die vorbereitenden Arbeiten mit der Puppe stellen folglich einen zentralen Schritt des Arbeitsprozesses dar. Mittlerweile findet sich eine große Auswahl an menschlichen Handpuppen, die in Kleidung, Mimik und Hautfarbe variieren und somit der Anerkennung von Vielfalt (vgl. Prengel 2006) gerecht werden.

Entscheidend für ein gelingendes Puppet Interview ist die Natürlichkeit der Interviewer*innen im Umgang mit der Puppe, je sicherer sich diese mit der Puppe fühlen, desto authentischer wirken sie. Dazu benötigt die Puppe eine eigene Identität und Persönlichkeit, diese steckt implizit bereits in der Gestaltung der Puppe. Handelt es sich um eine Puppe mit menschlichen Zügen, dann legt bereits die Kleidung Interpretationen zur Zuordnung der Geschlechtsidentität (vgl. Bachmann 2008) sowie zu ersten Einschätzung eines Charakters nahe. Dies muss im Forschungsprozess durchaus mit bedacht und kritisch reflektiert werden. Aus vielfaltssensibler Perspektive (vgl. Prengel 2006; Kölsch-Bunzen, Morys \& Koblauch 2015) kann eine Handpuppe in Form eines Tieres gewählt werden, die menschliche Eigenschaften erhält und ebenso im Rollenspiel für Kinder funktioniert. Nachteil ist aktuell noch, dass diese Puppen meist kleiner als die menschlich gestalteten Handpuppen sind. Zu beachten ist dabei, dass auch Tierfiguren von Kindern unterschiedlich emotional besetzt sind.

Nach dem ersten Eindruck der Passung muss die Handpuppe als Person ausgebaut und mit einer konkreten biografischen Geschichte versehen werden. Auf diesen Schritt verweist auch die etablierte Praxis der persona dolls im Ansatz Kinderwelten (vgl. Wagner 2008), die intensiv mit Puppen zur vorurteilsbewussten Bildung und Erziehung in Kindertagesstätten arbeiten (vgl. Beutel 2012; Azun 2011; Enßlin 2007; Krause 2007). Im Ansatz Kinderwelten (vgl. Wagner 2008) werden die Puppen in speziellen Fortbildungen durch die Pädagog*in selbst hergestellt und authentisch gestaltet. In der pädagogischen Arbeit mit den persona dolls erzählt die Puppe der Pädagog*in von eigenen Erlebnissen, die die Kinder zu Empathie, zum Nachdenken und zum darüber Sprechen anregen (Beutel 2012, 21).

\subsection{Puppet Interview mit einem semi-strukturierten Leitfaden}

Das Puppet Interview in Form eines semi-strukturierten Leitfadeninterviews anzulegen, wird aufgrund seiner rahmengebenden Form für die Altersgruppe empfohlen. Weitgehender Konsens besteht in der Kindheitsforschung, dass beispielsweise ein narratives Interview, das nur den ersten Impuls setzt, eine Überfor- 
derung für die Zielgruppe darstellt (vgl. Mey 2003; Vogl 2015). Auch vermittelt das Leitfadeninterview den Forschenden selbst die benötigte Sicherheit durch eine leitende Struktur und Vergleichbarkeit. Allerdings kann sie leicht zu einem Abfragestil und einem starren Abarbeiten des Leitfadens führen, der das Zustandekommen flüssiger Gespräche behindert (vgl. Zorn 2012; Vogl 2015). Eine so genannte Leitfadenbürokratie ist zu vermeiden, dennoch ist davon auszugehen, dass sich narrative Passagen, aber auch Stellen mit engen Fragen der interviewenden Person und dadurch knappe Antworten der Kinder wiederfinden lassen. Hier scheint ein Wechsel sinnvoll, da engere Fragen einfacher zu beantworten sind und somit eine entlastende Funktion haben können.

Generell führen erzählgenerierende Fragen, die für die Themen der Kinder allseits offen bleiben, zu mehr Erkenntnisgewinn als eng-strukturierende. Die Interviewpartner*innen haben die Freiheit, das Gespräch zu strukturieren und die ihnen bedeutsamen Sinngehalte ausführen (vgl. Przyborski \& Wohlrab-Sahr 2014). An die Fragen und Themen der Kinder anzuschließen und in einem Dialog zu bleiben (vgl. Alemzadeh 2014) stellt hohe Anforderungen an die Interviewenden. Nicht immer können Erwachsene an den Erfahrungsraum des Kindes anschließen, die kindspezifischen Deutungsmuster sind oft nicht unmittelbar zugänglich (vgl. Hülst 2012; Fuhs 2012). Zentral ist daher, einen Rahmen in Form von wenigen zentralen Leitfragen als Sicherheit aufzuspannen und darin dem Kind aber möglichst viele Optionen zu bieten, sich vielfältig auszudrücken (Fuhs \& Schneider 2012; Nentwig-Gesemann et al. 2017).

\section{Reflexion der Handpuppe als Mediator*in in den Puppet Interviews}

\subsection{Reduktion von Asymmetrien durch die persona Handpuppe}

Kindheitssoziologisch muss die Position von Kindern zu Erwachsenen unter Berücksichtigung des Konzeptes der generationalen Ordnung (vgl. Hengst \& Zeiher 2005; Bühler-Niederberger 2005; Kränzl-Nagl \& Mierendorff 2007) betrachtet werden. Die Zuordnung zu Generationen über Lebensalter ist nach Kränzl-Nagl \& Mierendorff (2007) keine natürliche Ordnung, sondern eine gesellschaftliche Konstruktion, wobei die Kategorien Kinder und Erwachsene in der sozialen Praxis beständig reproduziert werden (vgl. ebd.). Gerade in Interviews mit Kindern in Kontexten der institutionellen Bildung, Erziehung und Betreuung muss die Herstellung des Generationsunterschiedes und der damit verbundenen Asymmetrien bedacht werden. Forschende Praktiker*innen der Institution als auch externe Forscher*innen, die nicht als pädagogische Fachkräfte auftreten, sind Erwachsene und stehen damit im strukturell-hierarchischen Ungleichgewicht zu Kindern. 
Das Zwischenschalten der Puppe reduziert die Wahrnehmung einer strukturellen Überlegenheit der Erwachsenen. Die Situation einer qualitativen Befragung ist eine meist ungewohnte Situation, durch die Puppe als Mediator*in wird ein vertrautes Element eingeführt und die Situation erhält damit den Spielcharakter eines Rollenspiels ohne ihr gleichzeitig die Ernsthaftigkeit und Relevanz zu nehmen. Die Puppe nimmt der Situation das Ungewohnte, lässt es vertraut werden und eröffnet den Raum für vielfältige Ausdrucksmöglichkeiten. Den Kindern ist faktisch klar, dass die Interviewer*in durch die Puppe spricht, dennoch ist die Ansprechpartner*in auf der Spielebene eine Puppe. Hier zeigt sich, dass Kinder schnell einen Zugang zu der Puppe finden (vgl. Lampert 2000; Weise 2012) und Hemmungen abgebaut werden. Das Handpuppen-Interview stellt damit eine adäquate Möglichkeit dar, die „symbolische Gewalt“ (Hülst 2012, 71) und die Asymmetrien zwischen dem Erwachsenen und dem Kind zu verringern. Ängste, Hemmungen und sozial erwartetes Antwortverhalten der Kinder können ebenfalls vermindert werden (vgl. Paus-Haase 1998; Lampert 2000; Weise 2008).

\subsection{Sprachliche Ausdrucksweise und sprachliche Brüche durch die persona Handpuppe}

Bedenken bei Interviews mit Kindern herrschen gerade im Hinblick auf sprachliche Kohärenz und sprachliches Ausdrucksvermögen. Hierbei zeigt sich in Untersuchungen von Hausendorf (2001) als auch nach theoretischen Überlegungen von Hülst (2012), dass die sprachliche Kompetenzwahrnehmung damit zusammenhängt, ob Erwachsene die Kommunikation von Kindern als eigenständige Ausdrucksweisen anerkennen (vgl. Nentwig-Gesemann 2013, Nentwig-Gesemann et al. 2017; Mey 2003; Clark 2017) oder ihnen Defizite suggerieren und an „erwachsenen-zentrierten Kommunikationsgewohnheiten und Normalitätsunterstellungen "(Hülst 2012, 65) festhalten.

Interviewende, die eine Handpuppe als Mediator*in verwenden, schaffen einen Raum in dem sich Fiktion und Realität, Spielsituation und Realsituation nicht gegenüber stehen, sondern integriert und zusammengehörig betrachtet werden. Die Puppen werden nicht durch die Kinder selbst animiert, in Ansätzen kann dennoch von einem intermediären Raum - einem „Ermöglichungsraum “ (Fooken 2012, 38) - ausgegangen werden, in dem Utopien, Fantasien, Vertrautes und Neues stattfinden können (vgl. ebd.). Da im Vordergrund die subjektiven Deutungsstrukturen stehen, geben gerade Inszenierungen und Utopien Informationen über Bedeutsames und entkräften somit in diesem qualitativen rekonstruktiven Zugang die Bedenken der Verzerrung von objektiver Wahrheit.

Die Puppe eröffnet damit dem Kind durch das „So-tun-als-ob“ Möglichkeiten sich performativ auszudrücken und ins Rollenspiel zu wechseln. Darüber hinaus sind auch für die Interviewenden Inszenierungen auf der performativen Ebene über das Eintauchen in die Rolle der Puppe möglich, die dem Erwachsenen even- 
tuell sonst in seiner Rolle nicht zur Verfügung stehen würden. Die persona Handpuppe hat zudem den Vorteil, dass es in der Spielsituation bestimmte Sachverhalte erfragen kann, von denen auszugehen ist, dass eine erwachsene Interviewerin oder ein erwachsener Interviewer die Antwort kennt.

Gleichzeitig sind aber mit der Anwesenheit der Puppe in persona der Handpuppe methodische Herausforderungen und Verzerrungen kritisch zu beachten. Dem Kind steht mit der Puppe quasi eine weitere Person zur Verfügung, der es sich situationsbezogen zuwenden kann. Der Entfaltung eines eigenen Charakters der Puppe wird in der Vorbereitungsphase eine hohe Bedeutung beigemessen, dennoch kann diese Trennung nicht während des gesamten Interviews aufrechterhalten werden. Es entstehen Brüche, denn der Erwachsene kann in die Rolle eintauchen, aber er kann nicht darin aufgehen - er bleibt ein Erwachsener. Die erwachsenen Forschenden können folglich immer nur versuchen, sich der Perspektive des Kindes anzunähern, können sie aber nicht übernehmen und auch über die Handpuppe keine reale Peer-Interaktion herstellen.

Folglich ist die Frage zu stellen, ob die Trennung der Personen für das Gelingen der Methode notwendig ist. Kinder realisieren, dass es sich bei der persona Handрирре um die Interviewer*in handelt. Sie erkennen die Brüche und versuchen in der „Puppen-Spiel-Ebene“ zu bleiben, funktioniert dies nicht, zeigen sie sich sprachlich und kognitiv kompetent, die Ebenen zu wechseln bzw. wechseln situativ auch bewusst die Ebenen um Themen mit den jeweils richtigen Ansprechpartner*innen zu besprechen. Entscheidend für den Erfolg ist insgesamt, ob das jeweilige Kind sich auf dieses Spiel einlässt.

\subsection{Mitfühlende Kommunikation als Notwendigkeit der persona Handpuppe}

Die Forschenden wollen sich den subjektiven Deutungsmustern der Kinder annähern, können sich folglich nicht aus der Kommunikationssituation herausnehmen. Indem auf alltägliche Methoden wie das Gespräch zurückgegriffen wird, ist ein direkter Kontakt mit den Kindern hergestellt. Die Forscherin oder der Forscher bewegt sich zwischen den beiden Polen „Vertrautheit und Fremdheit“ (Hülst 2012, 72), in der kommunikativen Forschungssituation bedarf es der sozialen Nähe, während in der Analyse Distanz gefordert ist, um nicht Vorverständnisse den Daten überzustülpen und datengestützt „vom Vorwissen zum Wissen“ (Krotz 2005, 130) zu gelangen. Für die Durchführung der qualitativen Interviews wird daher eine "neutrale bis weiche Kommunikationstechnik“ (Lamnek 2010, 343) angewandt. Im Zentrum steht die berechtigte Forderung von nicht wertenden und damit das Gespräch zu stark verzerrenden Forschenden.

In Interviews mit Kindern scheint das allerdings eine schwer erfüllbare Empfehlung. Kinder fordern die Positionierung ein, sie brauchen einen „empathischen Zugang der Erwachsenen“ (Vogl 2015, 100; vgl. Remsperger 2010), der auch eine Bestätigung und Begeisterung beinhalten kann und somit sicherlich in seinen 
Aussagen auch Wertungen enthält, die es gilt methodisch im Sinne des „kontrolliert methodischen Fremdverstehens" (Bohnsack 2005, 68) und der Rekonstruktion aufzuarbeiten. Fragefehler durch Propositionen und Suggestionen lassen sich aufgrund der Komplexität des Verhältnisses zwischen Kindern und Erwachsenen als auch aufgrund des Gesprächsduktus nie ganz vermeiden. Je jünger die Kinder, so zeigte es sich im Material, desto eher fordern sie eine aufmunternde Bestärkung zum Gespräch in Form von Hörerrückmeldungen von der interviewenden Person ein. Dies könnte als Spezifikum von Interviews mit Kindern begriffen werden. Hegner formuliert provokativ im Sinne einer ,verwundbaren Beobachter_in mehr Mut zur Nähe im Feld“ (Hegner 2013, 13). Der Begriff geht dabei über die Idee von Sympathie hinaus, sich mitfühlend zu machen bedeutet, sich in die Vorstellungen des Gegenübers einfühlen, ohne notwendig deren „Gefühle und Gedankengänge zu übernehmen" (Hegner 2013, 13).

Für Dialoge mit Kindern formulieren Pädagog*innen das Desiderat des aufmerksamen, aktiven Zuhörens (vgl. Remsperger 2010), welches sich durch Kriterien wie ein körperlich aktives Zugewandtsein, ein reales Interesse an dem Gegenüber und ein Zurücknehmen der eigenen Person auszeichnet (vgl. ebd.). Techniken wie Paraphrase, Spiegeln oder Verbalisieren zeigen sich gerade für Interviews mit Kindern besonders sinnvoll. Bilanzierend kann nach Auffassung der Autorin formuliert werden, dass die Interviewführung mit Kindern eine in diesem Sinne mitfühlende, leidenschaftlich-anteilnehmende Forschungsperson benötigt, die aktiv zuhören kann, sich aber auch traut durch eigene Positionierungen und Hörerrückmeldungen das Gespräch mit dem Kind zu führen und aufrecht zu erhalten. Notwendig ist jedoch in Analyse und Auswertung den Beitrag zur Deutungskonstruktion systematisch mittels sequentieller rekonstruktiver Auswertungsmethoden (vgl. Bohnsack, Nentwig-Gesemann \& Nohl 2013) einzubeziehen, um so das Wechselverhältnis zwischen Nähe und Distanz differenziert auszuloten.

\section{Das Puppet Interview als Methode der Beteiligung für forschende Praktiker*innen - ein Ausblick}

Kinder haben ein Recht auf freie Meinungsäußerung und darauf in ihren Belangen gehört und als Expert*innen anerkannt zu werden (vgl. BMFJS 2014). Die UN-Kinderrechtskonvention sowie das Kinder- und Jugendhilfegesetz (\$8 SGB VIII) legen rechtlich fest, dass bereits in Institutionen früher Bildung pädagogische Fachkräfte verpflichtet sind, Kinder an Entscheidungen, die ihr Leben oder das Leben der Gemeinschaft betreffen, zu beteiligen (vgl. Hansen 2013, Knauer \& Sturzenhecker 2016). Folglich ist es aus kinderrechtstheoretischer Perspektive einerseits geboten Kinder in Forschung, der sie betreffenden Themenbereiche einzubinden (vgl. Gerarts 2017). Darüber hinaus erweisen sich Methoden wie das 
Puppet Interview auch für forschende Praktiker*innen (vgl. Nentwig-Gesemann, Nicolai 2008; Rinaldi 2006) als relevant, die vor dem Hintergrund einer demokratischen Bildung (vgl. Knauer \& Sturzenhecker 2016; Ruppin 2018) Kindern Möglichkeiten und Räume schaffen, sich für die eigenen Belange und die der Gemeinschaft einsetzen zu können sowie eigene Interessen als auch empathisch die von Anderen vertreten zu können (vgl. Hansen 2013). Hierfür - und dies zeigt der Ansatz der persona dolls aus dem Ansatz Kinderwelten (vgl. Wagner 2008) - eignen sich Puppen und hier die Puppet Interviews in besonderer Weise als Mittlerinstrumente.

Das Setting einer Befragung mittels Handpuppe ist dennoch eine ungewohnte Situation, kann aber von den an der Forschung beteiligten Kindern als Ausdruck von Bedeutsamkeit, Wertschätzung und Teilhabe interpretiert werden. Die Puppet Interview Methode bietet folglich gerade auch Kindern Chancen sich zu äußern, die ggf. in ihren (institutionellen) Kontexten wenig gehört werden oder die weniger Chancen haben sich auszudrücken, sei es aufgrund von Zuschreibungen oder auch faktischer sprachlicher Hürden oder aufgrund ihres Standings in der Peergroup.

Für folgende Projekte könnte es spannend sein auszuloten, inwieweit sich die Methode für die inklusive Forschung mit Kindern in besonders vulnerablen Konstellationen eignet (vgl. Gerarts 2017; Andresen et al. 2015). Denn tendenziell steht in Puppet-Interviews die Verbalsprache im Vordergrund, daher gilt die Prämisse möglichst verschiedene Methoden zu kombinieren, um der Heterogenität der Kinder und ihrer Ausdruckweisen in Ansätzen gerecht zu werden (vgl. Weise, Lempp \& Morys in diesem Band). Dabei ist gleichzeitig zu berücksichtigen, dass im Verständnis „von heterogen als unbegreiflich und unsagbar“ (Prengel 2010, 5) Ergebnisse in der Forschung mit Kindern als vorläufige Interpretationen zu betrachten sind und es „relevante Aspekte der Kinder gibt, mit denen wir arbeiten oder über die wir forschen, die aber unsere Erkenntnisse nicht erfasst haben." (Prengel 2010,5)

\section{Literatur}

Alemzadeh, M. (2014): Interaktionen im frühpädagogischen Feld. Ethnographische Bildungsforschung zu Interaktions- und Spielprozessen und deren Bedeutung für eine Didaktik der frühen Kindheit am Beispiel der Lernwerkstatt Natur. Universität zu Köln. Online unter: http://kups. ub.uni-koeln.de/5744/ (09.02.2019).

Andresen, S., Koch, C. \& König, J. (2015): Vulnerable Kinder. Interdisziplinäre Annäherungen. Wiesbaden.

Azun, S. (2011): Persona Dolls - Mit Kindern über Vielfalt sprechen, in: Welt des Kindes /2011. Online unter: https://situationsansatz.delfiles/texte\%20istalfachstelle_kinderwelten/kiwe_pdflAzun, \%20 Serap_PesonaDolls_Mit\%20Kindern\%20ueber\%20Vielfalt\%20sprechen_In\%20Welt\%20des\%20 Kindes\%202011.pdf(22.02.2019)

Bachmann, C. (2008): Kleidung und Geschlecht : Ethnographische Erkundungen einer Alltagspraxis. Online unter: https://doi.org/10.25595/226 (18.01.2019). 
Balluseck, H. von (Hrsg.)(2008): Professionalisierung der Frühpädagogik. Perspektiven, Entwicklungen, Herausforderungen. Opladen \& Farmington Hills.

Betz, T. \& Bischoff, S. (2013): Risikokind und Risiko Kind. Konstruktionen von Risiken in politischen Berichten. In: Kelle, H. \& Mierendorff, J. (Hrsg.): Normierung und Normalisierung der Kindheit. Weinheim, 60-81.

Betz, T. \& Eßer, F. (2016): Kinder als Akteure - Forschungsbezogene Implikationen des erfolgreichen Agency-Konzepts. Online unter: https://nbn-resolving.org/urn:nbn:de:0168-ssoar-48445-7 (12.11.2018).

Beutel, P. (2012): Persona Dolls. Puppen mit Persönlichkeit besuchen die Kindergruppe. In: Theorie und Praxis der Sozialpädagogik, H.8, 20-23.

Bohnsack, R. (2005): Standards nicht-standardisierter Forschung in den Erziehungs- und Sozialwissenschaften. In: Zeitschrift für Erziehungswissenschaft 8 (4), 63-81.

Bohnsack, R., Nentwig-Gesemann, I. \& Nohl, A.-M. (2013): Die dokumentarische Methode und ihre Forschungspraxis. Grundlagen qualitativer Sozialforschung. Wiesbaden.

Bühler-Niederberger, D. (2005): Kindheit und die Ordnung der Verhältnisse. München.

Bundesministerium für Familie, Senioren, Frauen und Jugend (2014): UN-Kinderrechtskonvention im Wortlaut mit Materialien. Übereinkommen über die Rechte des Kindes. Online unter: https:// www.netzwerk-kinderrechte.delfileadmin/bilder/user_upload/\%C3\%9Cbereinkommen-\%C3\%BCber-die-Rechte-des-Kindes_2014.pdf(28.01.2019).

Clark, A. (2017): Listening to young children. A guide to Understanding and Using the Mosaic Approach. London \& Philadelphia.

Eide, B.J. \& Winger, N. (2005): From the children's point of view: methodological and ethical challenges. In: Clark, A., Kjorholt, T. \& Moss, P. (Hrsg.): Beyond Listening: children's perspectives on early childhood services. University of Bristol, 71-90.

Enßlin, U. (2007): Alle Kinder sind gleich - jedes Kind ist besonders. Kommunikation mit Kindern vorurteilsbewusst gestalten. In: Theorie und Praxis der Sozialpädagogik, H.3, 28-31.

Epstein, I., Stevens, B., McKeever, P., Baruchel, S. \& Jones, H. (2008): Using puppetry to elicit children's talk for research. In: Nursing inquiry, 15, H.1, 49-56.

Fooken, I. (2012): Wider die "Puppenstarre«. Ein Plädoyer für die (Wieder-)Aneignung des intermediären und imaginären Raums im Spiel mit Puppen. In: Schröteler-von Brandt, H., Coelen, T. \& Zeising, A. (Hrsg.): Raum für Bildung: Ästhetik und Architektur von Lern- und Lebensorten. Bielefeld, 33-42.

Fuhs, B. (2012): Kinder im qualitativen Interview - zur Erforschung subjektiver kindlicher Lebenswelten. In: Heinzel, F. (Hrsg.): Methoden der Kindheitsforschung. Weinheim, 80-103.

Fuhs, B. \& Schneider, S. (2012): Normalisierungsvorstellungen und Adultismus als Probleme für die erzählerische Erschließung frühkindlicher Lebenswelten. In: Frühe Bildung, 1, H.3, 125-130.

Gauda, G. (2001): Theorie und Praxis des therapeutischen Puppenspiels. Lebendige Psychologie C. G. Jungs. Dortmund.

Gerarts, K. (2017): Inklusive Forschung über Kinder mit Kindern. In: Platte, A. \& Amirpur, D. (Hrsg.): Handbuch Inklusive Kindheiten. Opladen \& Toronto, 131-144.

Groundwater-Smith, S., Dockett, S. \& Bottrell, D. (2014): Participatory research with children and young people. London.

Hansen, R. (2013): Mitbestimmung der Kleinsten im Kita-Alltag - so klappt's! In: KiTa aktuell ND Ausgabe 03.2013, 67-69.

Hausendorf, H. (2001): Was ist „altersgemäßes“ Sprechen? Empirische Anmerkungen am Beispiel des Erzählens und Zuhörens zwischen Kindern und Erwachsenen. In: Osnabrücker Beiträge zur Sprachtheorie, H.62, 11-33.

Hegner, V. (2013): Vom Feld verführt. Methodische Gratwanderungen in der Ethnografie. Online unter: http://www.qualitative-research.net/index.php/fqs/article/viewFile/1957/3597 (09. 01.2019). 
Heinzel, F. (Hrsg.) (2012): Methoden der Kindheitsforschung: Ein Überblick über Forschungszugänge zur kindlichen Perspektive. Weinheim \& Basel.

Hengst, H. \& Zeiher H. (Hrsg.)(2005): Kindheit soziologisch. Wiesbaden.

Honig, M.-S. (1999): Forschung „,vom Kinde aus“? Perspektivität in der Kindheitsforschung. In: Honig, M.-S., Lange, A. \& Leu, H.R. (Hrsg.): Aus der Perspektive von Kindern? Zur Methodologie der Kindheitsforschung. Weinheim \& München, 33-50.

Honig, M.-S. (2009): Ordnungen der Kindheit - Problemstellungen und Perspektiven der Kindheitsforschung. Weinheim \& München.

Honig, M.-S., Lange, A. \& Leu, H.R. (Hrsg.)(1999): Aus der Perspektive von Kindern? Zur Methodologie der Kindheitsforschung. Weinheim \& München.

Hülst, D. (2012): Das wissenschaftliche Verstehen von Kindern. In: Heinzel, F. (Hrsg.): Methoden der Kindheitsforschung: Ein Überblick über Forschungszugänge zur kindlichen Perspektive. Weinheim \& Basel, 52-79.

Kelle, H. \& Mierendorff, J. (Hrsg.)(2013): Normierung und Normalisierung der Kindheit. Weinheim.

Knauer, R. \& Sturzenhecker, B. (2016): Demokratische Partizipation von Kindern. Weinheim, Basel.

Kölsch-Bunzen, N., Morys, R. \& Knoblauch, C. (2015): Kulturelle Vielfalt annehmen und gestalten. Eine Handreichung zur Umsetzung des Orientierungsplans für Kindertageseinrichtungen in Baden-Württemberg. Freiburg im Breisgau.

Kränzl-Nagl, R. \& Mierendorff, J. (2007): Kindheit im Wandel. Annäherung an ein komplexes Phänomen. Online unter: http://www.sws-rundschau.at/archiv/SWS_2007_1_kraenzl-nagl_mierendorff. $p d f(09.01 .2018)$.

Krause, A. (2007): Magie mit Methode. Persona Dolls unterstützen Dialoge mit Kindern. In: Betrifft Kinder, H.3, 13-17.

Krotz, F. (2005): Neue Theorien entwickeln: Eine Einführung in die Grounded Theory, die Heuristische Sozialforschung und die Ethnographie anhand von Beispielen aus der Kommunikationsforschung. Köln.

Lamnek, S. (2010): Qualitative Sozialforschung: Lehrbuch. Weinheim und Basel.

Lampert, C. (2000): Spannung, Spiel und Schokolade. Aspekte qualitativer Forschungsmethoden mit Vorschulkindern am Beispiel einer Untersuchung zum Verständnis von Fernsehwerbung. In: Paus-Haase, I. \& Schorb, B. (Hrsg.): Qualitative Kinder- und Jugendmedienforschung. Theorien und Methoden: ein Arbeitsbuch. München, 115-129.

Lothar, M. \& Wegener, C. (Hrsg.)(2005): Qualitative Medienforschung. Ein Handbuch. Konstanz.

Marci-Boehncke, G. \& Rath, Matthias (2014): Action Research reloaded: Grounded Practice Warum Netzwerkprojekte zur Kooperativen Medienbildungsverantwortung die Interventionsforschung brauchen. In: Hartung, A., Schorb, B., Niesyto, H., Moser, H. \& Grell, P. (Hrsg.): Methodologie und Methoden medienpädagogischer Forschung (Jahrbuch Medienpädagogik 10). Wiesbaden, 231-251.

Measelle, J.R., Ablow, J.C., Cowan, P.A. \& Cowan, C.P. (1998): Assessing Young Children's Views of Their Academic, Social, and Emotional Lives: An Evaluation of the Self-Perception Scales of the Berkeley Puppet Interview. Online unter: https://pages.uoregon.eduldslab/Papers_files/1998\%20 Child\%20Devo\%20Measelle\%20et\%20al.pdf(11.01.2019).

Mey, G. (2003): Zugänge zur kindlichen Perspektive: Methoden der Kindheitsforschung (Forschungsbericht aus der Abteilung Psychologie im Institut für Sozialwissenschaften, 1-2003). Berlin: Technische Universität Berlin, Institut für Sozialwissenschaften, Abt. Psychologie. Online unter: http:// hdl.handle.net/20.500.11780/917 (20.02.2019).

Nentwig-Gesemann, I. (2007): Forschende Haltung. Professionelle Schlüsselkompetenz von FrühpädagogInnen. In: Sozial Extra, 31, H.5/6, 20-22. 
Nentwig-Gesemann, I. (2013): Qualitative Methoden der Kindheitsforschung. In: Stamm, M. \& Edelmann D. (Hrsg.): Frühkindliche Bildungsforschung. Wiesbaden: 759-770.

Nentwig-Gesemann, I. \& Nicolai, K. (2008): Praktische, theoretische und persönliche Annäherungen an das forschende Lernen. In: Daiber, B. \& Weiland, I. (Hrsg.): Impulse der Elementardidaktik. Eine gemeinsame Ausbildung für Kindergarten und Grundschule. Baltmannsweiler, 117-128.

Nentwig-Gesemann, I. \& Nicolai, K. (2008): Rekonstruktive Forschung in der Frühpädagogik. In: von Balluseck, H. (Hrsg.): Professionalisierung der Frühpädagogik. Perspektiven, Entwicklungen, Herausforderungen. Opladen \& Farmington Hills, 251-265.

Nentwig-Gesemann, I., Walther, B. \& Thedinga, M. (2017): Kita-Qualität aus Kindersicht. Eine Studie des DESI-Instituts der Deutschen Kinder- und Jugendstiftung. Online unter: https:// www.qualitaet-vor-ort.org/wp-content/uploads/2017/03/17.03.2017_Zusammenfassung_QuaKi.pdf (21.02.2019).

Oerter, R. (1999): Psychologie des Spiels: Ein handlungstheoretischer Ansatz. Weinheim und Basel.

Paus-Haase, I. (1998): Heldenbilder im Fernsehen. Eine Untersuchung zur Symbolik von Serienfavoriten in Kindergarten, Peer-Group und Kinderfreundschaften. Wiesbaden.

Paus-Haase, I. \& Schorb, B. (Hrsg.)(2000): Qualitative Kinder- und Jugendmedienforschung. Theorien und Methoden: ein Arbeitsbuch. München.

Paus-Hasebrink, I. (2017, 2005): Forschung mit Kindern und Jugendlichen. In: Lothar M. \& Wegener, C. (Hrsg.): Qualitative Medienforschung. Ein Handbuch. Konstanz, 276-282.

Perren, S. \& Klitzing, K. von (2008): Untersuchung von Kindergartenkindern mit einem Puppeninterview: Bedeutsamkeit und Anwendung. In: Kinder- und Jugendmedizin, 8, H.1, 25-30.

Perren, S., Stadelmann, S. \& Klitzing, K. von (2009): Child and family characteristics as risk factors for peer victimization in kindergarten. In: Schweizerische Zeitschrift für Bildungswissenschaften, $31,1,13-32$.

Phelan, S. K. \& Kinsella, E. A. (2013): Picture This ... Safety, Dignity, and Voice - Ethical Research With Children: Practical Considerations for the Reflexive Researcher. Qualitative Inquiry, 19, H.2, 81-90.

Pohl, G. (2014): Kindheit - aufs Spiel gesetzt: Vom Wert des Spielens für die Entwicklung des Kindes. Berlin \& Heidelberg.

Powell, M.A., Graham, A. \& Julia Truscott, (2016): Ethical research involving children: facilitating reflexive engagement.

Prengel, A. (2006): Pädagogik der Vielfalt: Verschiedenheit und Gleichberechtigung in Interkultureller, Feministischer und Integrativer Pädagogik. 3. Auflage. Wiesbaden.

Prengel, A. (2010): Von einer Ausländerpädagogik zur inklusiven Frühpädagogik - Neue Anforderungen an frühpädagogische Fachkräfte. WiFF Fachforum: 29.06.2010 - München Online https:// www.weiterbildungsinitiative.delfleadmin/_migrated/content_uploads/WiFF_Fachforum_Inklusion_ Impulsreferat_Prof_Dr_Prengel.pdf(31.01.2019).

Przyborski, A. \& Wohlrab-Sahr, M. (2014): Qualitative Sozialforschung. München.

Punch, S. (2002): Research with Children. The Same or Different from Research with Adults? Online unter: https://journals.sagepub.com/doi/pdfl10.1177/0907568202009003005 (18.01.2019).

Remsperger, R. (2010): Sensitive Responsivität: Zur Qualität pädagogischen Handelns im Kindergarten. Wiesbaden.

Rinaldi, C. (2006): In Dialogue with Reggio Emilia. Listening, researching and learning. London \& New York.

Roth, J.H., Dadds, M.R. \& McAloon, J. (2004): Evaluation of a Puppet Interview to Measure Young Children's Self-Reports of Temperament. In: Behaviour Change, 21, H.1, 37-56.

Roux, S. (2002): Wie sehen Kinder ihren Kindergarten? Theoretische und empirische Befunde zur Qualität von Kindertagesstätten. Weinheim, München \& Heidelberg.

Ruppin, I. (2018): Kinder und Demokratie. Weinheim \& Basel. 
Schröteler-von Brandt, H., Coelen, T. \& Zeising, A. (Hrsg.) (2012): Raum für Bildung: Ästhetik und Architektur von Lern- und Lebensorten. Bielefeld.

Stamm, M. \& Edelmann D. (Hrsg.)(2013): Frühkindliche Bildungsforschung. Wiesbaden.

Trautmann, T. (2009): Interviews mit Kindern: Grundlagen, Techniken, Besonderheiten, Beispiele. Wiesbaden.

Vogl, S. (2015): Interviews mit Kindern führen: Eine praxisorientierte Einführung. Weinheim.

Wagner, P. (Hrsg.)(2008): Handbuch Kinderwelten: Vielfalt als Chance - Grundlagen einer vorurteilsbewussten Bildung und Erziehung. Freiburg, Basel \& Wien.

Weise, M. (2008): Der Kindergarten wird zum „Forschungsort“ - Das Puppet Interview als Forschungsmethode für die Frühe Bildung. Online unter: https://www.ph-ludwigsburg.delfileadmin/ subsites/1b-mpxx-t-O1/user_files/Online-Magazin/Ausgabe11/Weise11.pdf(12.01.2016).

Weise, M. (2012): Kinderstimmen: Eine methodologische Untersuchung zum multi-perspektivischen Erfassen kindlichen Mediennutzungsverhaltens und Medienerlebens - Ein Beitrag zur frühkindlichen Medienbildungsforschung. Dissertation, Technische Universität Dortmund. Online unter: https://eldorado.tu-dortmund.de/bitstream/2003/30136/1/Dissertation_30.11.2011_Weise_DruckfassungPublikation.pdf(20.02.2019)

Zorn, I. (2012): Konstruktionstätigkeit mit Digitalen Medien. Eine qualitative Studie als Beitrag zur Medienbildung. Fachbereich Erziehungs- und Bildungswissenschaften, Boizenburg. 